Rapid Reviews COVID-19

\title{
Review 1: "Inhaled budesonide in the treatment of early COVID- 19 illness: a randomised controlled trial"
}

Ying-Rong $\mathrm{Du}^{1}$, Hai-yan $\mathrm{Fu}^{1}$

${ }^{1}$ Dali University, sixth affilated hospital, China

Published on: Apr 26, 2021

DOI: $10.1162 / 2 \mathrm{e} 3983 f 5 . f 99 \mathrm{~d} 4 \mathrm{e} 65$

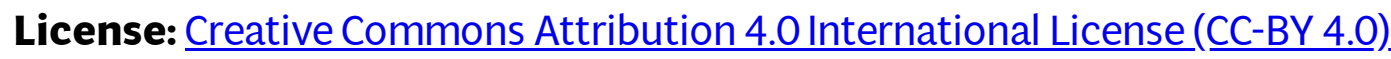




\section{$\underline{\text { RR:C19 Evidence Scale rating by reviewer: }}$}

- Reliable. The main study claims are generally justified by its methods and data. The results and conclusions are likely to be similar to the hypothetical ideal study. There are some minor caveats or limitations, but they would/do not change the major claims of the study. The study provides sufficient strength of evidence on its own that its main claims should be considered actionable, with some room for future revision.

$* * * * * * * * * * * * * * * * * * * * * * * * * * * * * * * * * * * * * * *$

\section{Review:}

Budesonide was commonly used with chronic respiratory disease, as a representative of glucocorticoids. It is currently clear that budesonide not only suppresses the immune response that occurs when asthma occurs but also has anti-inflammatory effects. Some research found that the high fatality and morbidity rates associated with COVID-19 can be partly attributed to dysregulated immune response and cytokine storm. So, inferring theoretically, the methods and conclusions of this study are reasonable.

Everyone hopes that the pandemic will be effectively controlled, whether through the use of vaccines or pharmaceuticals. Therefore, our research results should be more objective and reasonable than ever. So, I present my opinion on this research. In this study, the primary end point was COVID-19-related urgent care visit, emergency department assessment, or hospitalization. The rationality of the primary study endpoint needs to be further considered. COVID-19 brings a heavy burden to the medical system and, in many cases, the rate of hospitalization is not solely due to illness but may depend on the capacity of the medical system.

In regards to the secondary outcomes (Self-reported clinical recovery), my personal understanding is that self-reported results would be the conclusion drawn by the patients outlining their own feelings, which are subjective, and such an outcome would be difficult to quality control. There are many factors that affect the outcome, such as knowledge of the disease, social status. and medical support, etc. If there is more objective data to supplement the conclusions, the results would be more convincing.

During a pandemic, any work that contributes to disease control and prevention is worth encouraging, so I personally think that the results of this study are gratifying. Of 
course, I hope that this research will be further improved and made more scientific, which would help in controlling the pandemic. 